\title{
Preimplantation valvuloplasty in transcatheter aortic valve replacement: To BAV or not to BAV?
}

\author{
Mani Arsalan, MD, , ${ }^{a, b}$ John J. Squiers, BSE, ${ }^{b}$ and Thomas Walther, MD, $\mathrm{PhD}^{\mathrm{a}}$ \\ From the ${ }^{\mathrm{a} D e p a r t m e n t ~ o f ~ C a r d i a c ~ S u r g e r y, ~ K e r c k h o f f ~ H e a r t c e n t e r, ~ B a d ~ N a u h e i m, ~ G e r m a n y ; ~ a n d ~}{ }^{\mathrm{b}}$ Baylor Research \\ Institute, Baylor Scott \& White Health, Dallas, Tex. \\ Disclosures: Authors have nothing to disclose with regard to commercial support. \\ Received for publication Aug 7, 2015; accepted for publication Aug 8, 2015. \\ Address for reprints: Thomas Walther, MD, PhD, Department of Cardiac Surgery, Kerckhoff Heartcenter, Bene- \\ kestrasse 2-8, 61231 Bad Nauheim, Germany (E-mail: t.walther@kerckhoff-klinik.de). \\ J Thorac Cardiovasc Surg 2015;150:1118-9 \\ $0022-5223 / \$ 36.00$ \\ Copyright (C) 2015 by The American Association for Thoracic Surgery \\ http://dx.doi.org/10.1016/j.jtcvs.2015.08.017
}

During the last decade, transcatheter aortic valve replacement (TAVR) has emerged as the treatment of choice of severe aortic stenosis for patients who are not operative candidates and the preferred alternative for patients at high risk. In the early years of TAVR, balloon aortic valvuloplasty (BAV) was routinely performed to "pave the way" for valve implantation. More recently this approach has been simplified by many operators, with good clinical outcomes. ${ }^{1}$

BAV by itself had previously been the only transcatheter treatment option available to reduce symptoms in patients with surgically inoperable aortic stenosis. Because of early restenosis, however, isolated BAV was not capable of prolonging survival. ${ }^{2}$ Instead, BAV has been incorporated into TAVR and is performed immediately before valve implantation. The dilatation of the native valve by BAV has 2 purposes: (1) to allow the delivery system, loaded with the bioprosthesis, to safely pass through the native calcified aortic valve; and (2) to allow full expansion of the implanted TAVR valve, in turn preventing paravalvular leak (PVL). For the latter reason alone, BAV will remain essential for TAVR performed with self-expanding devices. On the other hand, BAV may be superfluous when a balloon-expandable TAVR device is deployed. This hypothesis is of interest, because several studies have demonstrated that BAV may be associated with an increased risk of cerebral embolization. ${ }^{3}$ Omitting BAV thus could potentially reduce the stroke rate after TAVR. In exchange, the lack of an "opened" native valve might result in inferior valve positioning or expansion, which could result in an increased rate of PVL. This opposite, however, could just as well be the case.

In this issue of the Journal, Wong and colleagues ${ }^{4}$ report the impact of preimplantation BAV on outcomes of patients undergoing TAVR. They retrospectively analyzed the clinical outcomes of 121 patients undergoing transfemoral (TF) or transapical (TA) implantation of the SAPIEN valve (Edwards Lifesciences, Inc, Irvine, Calif). During all TA TAVR cases $(n=50)$, because the delivery device allows

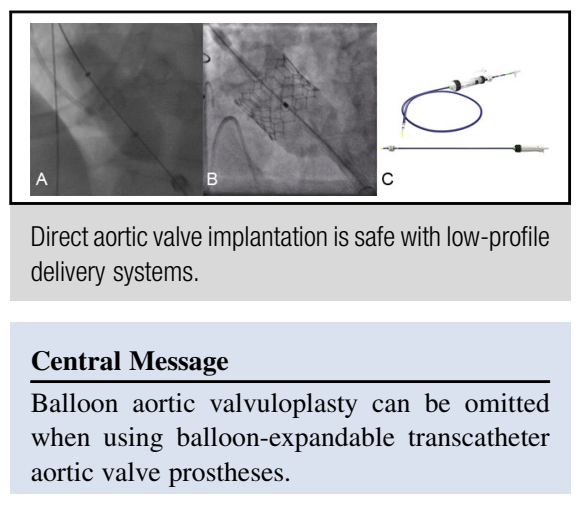

See Article page 1111.

easy crossing of the native valve, BAV was omitted. The TF TAVR cases were performed with BAV in every case.

The following are the noteworthy findings from the study of Wong and colleagues ${ }^{4}$ :

(1) TA TAVR without preimplantation BAV is feasible and safe, with no difference in 30-day mortality relative to TF-TAVR with preimplantation BAV.

(2) Omitting BAV did not increase the rate of PVL in the TA TAVR cohort.

(3) No difference in the rate of postdeployment balloon dilatation was observed between the groups.

(4) Omitting BAV did not decrease the stroke rate in the TA TAVR group relative to TF TAVR with preimplantation BAV.

In light of these findings, one might conclude that BAV is superfluous in TA TAVR with a balloon-expandable device. Even Wong and colleagues, ${ }^{4}$ however, admit that the study was underpowered to detect differences in PVL and stroke rate. Furthermore, differences in baseline characteristics between patients undergoing TAVR by the TF and TA routes, including rates of peripheral vascular disease, may have confounded the analyses. The different delivery routes used for each cohort may have also confounded the comparison.

In Europe, TAVR without BAV is routine practice for the implantation of the balloon-expandable SAPIEN XT and more recently, SAPIEN 3 prostheses. Conradi and colleagues ${ }^{5}$ compared the outcomes of 50 consecutive patients undergoing TA TAVR without BAV with those of 50 patients treated conventionally TA TAVR with preimplantation BAV. Although they reported a reduction in the use of 
contrast agent, Conradi and colleagues ${ }^{5}$ could not detect a reduced incidence of cerebrovascular events. Kempfert and colleagues ${ }^{6}$ enrolled 128 patients undergoing TA TAVR with the SAPIEN XT device, with 40 of the procedures $(31.2 \%)$ performed without BAV. Propensity scorebased pair matching was performed to adjust for baseline variables in this study. As in the previously mentioned reports, Kempfert and colleagues ${ }^{6}$ could not detect any reduction of cerebrovascular events after omitting BAV; however, fluoroscopy time was significantly lower in patients without BAV.

The existing literature confirms both the feasibility and safety of TA TAVR without preimplantation BAV. Whether omitting BAV truly reduces cerebrovascular events, however, remains unknown. Thus the question remains: To BAV or not to BAV? On the basis of current clinical experience, omitting BAV is feasible during the implantation of balloon-expandable valves through the retrograde TF approach or the antegrade TA approach. Improved patient screening (with perfect computed tomographically based sizing), large operator experience, and technically welldeveloped systems, including specific nose cones, together allow safe and routine procedures without preimplantation $\mathrm{BAV}$. In rare cases, secondary BAV can be performed as a bailout if required. Simplification of the TAVR procedure is an obvious advantage of omitting BAV. Further advantages, such as reduced hemodynamic compromise, lower stroke risk, and the potential for better valve sealing (and thus less PVL), will have to be proved by larger studies, ideally prospective, randomized trials.

\section{References}

1. Ben-Dor I, Pichard AD, Satler LF, Goldstein SA, Syed AI, Gaglia MA Jr, et al, Complications and outcome of balloon aortic valvuloplasty in high-risk or inoperable patients. JACC Cardiovasc Interv. 2010;3:1150-6.

2. Lieberman EB, Bashore TM, Hermiller JB, Wilson JS, Pieper KS, Keeler GP, et al. Balloon aortic valvuloplasty in adults: failure of procedure to improve long-term survival. J Am Coll Cardiol. 1995;26:1522-8.

3. Kahlert P, Al-Rashid F, Döttger P, Mori K, Plicht B, Wendt D, et al. Cerebral embolization during transcatheter aortic valve implantation: a transcranial Doppler study. Circulation. 2012;126:1245-55.

4. Wong SC, Pawar S, Minutello RM, Horn EM, Skubas NJ, Devereux RB et al. Device success and 30-day clinical outcome in patients undergoing preimplant valvuloplasty in transfemoral versus omitting valvuloplasty in transapical transcatheter aortic valve replacement. J Thorac Cardiovasc Surg. 2015; 150:1111-7.

5. Conradi L, Seiffert M, Schirmer J, Koschyk D, Blankenberg S, Reichenspurner H, et al. Transapical transcatheter aortic valve implantation without prior balloon aortic valvuloplasty: feasible and safe. Eur J Cardiothorac Surg. 2014;46:61-6.

6. Kempfert J, Meyer A, Kim WK, van Linden A, Arsalan M, Blumenstein J, et al First experience without pre-ballooning in transapical aortic valve implantation: a propensity score-matched analysis. Eur J Cardiothorac Surg. 2015;47:31-8; discussion 38 . 